\title{
Uso de hábitats modificados por aves dependientes de bosque tropical en la región caribeña de Guatemala
}

\author{
Alexis Cerezo $^{1,2}$, Chandler S. Robbins ${ }^{3} \&$ Barbara Dowell $^{3}$ \\ 1. Fundación para el Ecodesarrollo y la Conservación (FUNDAECO), 25 calle 2-39, zona 1, Ciudad de Guatemala, \\ Guatemala, CP 0101; acerezo@agro.uba.ar \\ 2. Cátedra de Métodos Cuantitativos Aplicados, Facultad de Agronomía, Universidad de Buenos Aires, Av. San Martín \\ 4453, (1417) C.A.B.A., Argentina. \\ 3. Patuxent Wildlife Research Center, Biological Division of the United States Geological Survey, U.S.A. 11410 \\ American Holly Drive, Laurel, MD 20708-4015; crobbins@usgs.gov, bdowell@usgs.gov
}

Recibido 27-III-2008. Corregido 09-VI-2008. Aceptado 09-VII-2008.

\begin{abstract}
Modified-habitat use by tropical forest-dependent birds in the Caribbean region of Guatemala. As natural areas are reduced into isolated remnants, the importance of secondary habitats for species conservation will increase. Consequently, the conservation value of human-modified or created secondary habitats must be determined. In this study, we evaluated the conservation potential of three habitats associated to cattle ranching (riparian forest, live fence, and pasture) for tropical forest birds in the Caribbean region of Guatemala. We studied the bird communities of five tropical forest and riparian forest (henceforth river) sites, three live fence sites and four pasture sites, in five cattle ranches in the region, and compared the four habitats based on the following parameters: species richness, number of individuals, species composition and an index of conservation importance. A 10-point series was sampled (six repetitions of each series) in each habitat site using the point-count method, in march and april of 1998 and 1999. Although forest, river and live fence had similar mean species numbers, considering all species (111, 96 and 94 species, respectively), and river and live fence surpassed the forest in mean number of individuals (80 and 72 compared to 56 individuals), river and live fence had significantly fewer mean numbers of interior-forest resident species and individuals (56, 21 and 15 species, and 61, 19 and eight individuals in forest, river and live fence, respectively). River and live fence, when compared to forest, had no significant differences in the number of resident forest-edge species and individuals, but their edge-species composition was significantly different from forest. Those resident edge species that were relatively abundant in forest had very low abundances in the modified habitats, and those that were abundant in river and live fence were rare or absent in forest sites. With respect to migratory species, both river and live fence were very important, and the river was more important than forest, according to the conservation importance index. We conclude that these modified habitats have very little value for tropical forest-dependent resident species, but are extremely important for migratory species, particularly those of arboreal habits. We also stress that the conservation importance of alternative habitats should be based on species composition, as opposed to species richness and abundance, and defined as a function of species of particular conservation importance, in our locality, those dependent of tropical lowland forest. Rev. Biol. Trop. 57 (1-2): 401-419. Epub 2009 June 30.
\end{abstract}

Key words: habitat use, birds, tropical forest, riparian forest, live fence, pasture, conservation importance.

La destrucción y fragmentación de hábitat causada por la extracción de madera, las plantaciones forestales, los cultivos y la ganadería son las mayores amenazas para el mantenimiento de la diversidad biológica en los neotrópicos (Wilcove et al. 1985, Pimm et al.
1995, Pimm y Raven 2000). Las tasas más altas de deforestación se han registrado en México, Centroamérica y el Caribe (Kaimowitz 1996, Smith et al. 2001, FAO 2005). Sin embargo, la deforestación rara vez es total y/o permanente (FAO 1999, Harris y Pimm 2004). Los 
remanentes de bosque tropical se encuentran junto con diferentes hábitats que varían en su grado de complejidad vegetacional y similitud con el bosque original. Por lo tanto, a medida que las áreas de vegetación natural en los neotrópicos estén siendo reducidas a remanentes aislados, la importancia de la vegetación secundaria para la conservación de especies aumentará (Janzen 1988, Vandermeer y Perfecto 1997). Las opciones de manejo del paisaje que contribuyan a la conservación de especies y produzcan réditos económicos deben ser basados sobre una clara comprensión de qué diversidad biótica persiste en estos paisajes, y cómo la composición y estructura de la matriz agricultural influencian su potencial de conservación (Harvey et al. 2006).

Muchas aves neotropicales pueden adaptarse ecológicamente y a menudo aparecen en hábitats altamente modificados (Hutto 1992, Robbins et al. 1992, Stotz et al. 1996, Estrada et al. 1997, Petit et al. 1999, Daily et al. 2001). La capacidad de utilizar estos hábitats probablemente se deba a adaptaciones obtenidas por presiones de selección en el pasado, como aquellas provistas por perturbaciones frecuentes de hábitat o la existencia de bordes naturales (Estrada et al. 1997, Harris y Pimm 2004). Por lo tanto, las especies de bosque que tengan la capacidad de tolerar y sobrevivir en estos paisajes modificados probablemente sean las que perduren en el futuro.

En la región Caribeña de Guatemala, como en otras de Centroamérica (Kaimowitz 1996), el mayor impacto sobre el bosque tropical lluvioso ha provenido de la ganadería: entre 1976 y 1998 , aproximadamente el $50 \%$ del bosque a elevaciones menores a los $100 \mathrm{msnm}$ fue eliminado para el establecimiento de pasturas de forrajeo (Fundación para el Ecodesarrollo y la Conservación (FUNDAECO), datos no publicados). Estos establecimientos ganaderos se caracterizan por una variedad de hábitats, principalmente bosque continuo o remanentes de bosque que varían en su tamaño, bosques secundarios a orillas de ríos (bosque ribereño, de aquí en adelante "río"), cercos vivos y la pastura misma. A pesar de que otras investigaciones han estudiado el uso de hábitats en paisajes ganaderos por aves en otras localidades de Mesoamérica (Estrada et al. 1997, Daily et al. 2001, Hughes et al. 2002, Harvey et al. 2006), éstas no han considerado el valor de dichos hábitats para las especies típicas de bosque explícitamente, sino para todas las especies. Los objetivos de este estudio fueron evaluar el uso de cuatro hábitats en paisajes ganaderos por las aves residentes y migratorias dependientes de bosque tropical y el potencial de conservación de los últimos tres (bosque ribereño, cerco vivo y pastizal) para éstas. Evaluamos estas preguntas con respecto a la totalidad de especies, y explícitamente para las especies dependientes de bosque y de forma separada para las especies residentes y migratorias.

\section{MATERIALES Y MÉTODOS}

Area de estudio: El estudio se realizó dentro de la zonas de uso múltiple y de amortiguamiento de la Reserva Protectora de Manantiales Cerro San Gil, Izabal, Guatemala. Según el sistema Thornthwaite (1948), el clima es cálido con invierno benigno, sin estación seca bien definida. La precipitación media anual es de 3 $100 \mathrm{~mm}$, las temperaturas máximas y mínimas absolutas son de $36^{\circ} \mathrm{C}$ y $14^{\circ} \mathrm{C}$, respectivamente, y la temperatura media anual es de $26^{\circ} \mathrm{C}$. La humedad relativa media anual ha sido de $83 \%$ y la evapotranspiración media anual de 1,668 mm (INSIVUMEH 2005). La zona de vida (Holdridge 1970) es de bosque tropical muy húmedo (bmh-T).

El estudio se realizó en cinco establecimientos ganaderos (fincas): Linares (15 $43^{\prime}$ '58'

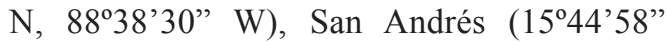

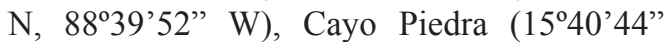
$\left.\mathrm{N}, 88^{\circ} 53^{\prime} 40^{\prime \prime} \mathrm{W}\right)$, Río Bonito (15\%41'14" $\left.\mathrm{N}, 88^{\circ} 52^{\prime} 28^{\prime \prime} \mathrm{W}\right)$ e Higuerito $\left(15^{\circ} 42^{\prime} 34^{\prime \prime} \mathrm{N}\right.$, $\left.88^{\circ} 51^{\prime} 41^{\prime \prime} \mathrm{W}\right)$.

Descripción de los hábitats evaluados: El bosque maduro (de aquí en adelante, bosque) varió en cuanto a su grado de complejidad y perturbación. En general, estuvo caracterizado por dos doseles arbóreos, el primero entre 
4 y $6 \mathrm{~m}$ de altura y el segundo entre 10 y $15 \mathrm{~m}$, con árboles emergentes entre 15 y 25 $\mathrm{m}$ de altura. Algunos géneros representativos de los estratos arbóreos fueron: Pouteria, Pithecolobium, Manilkara, Brosimum, Ceiba, Stemmadenia, Cecropia y Cymbopetalum. El sotobosque tuvo una altura entre 1 y $2.5 \mathrm{~m}$ y estuvo dominado por palmas (Arecaceae) de los géneros Calyptrogyne, Astrocaryum, Reinhardtia, Chamaedorea y Attalea.

La vegetación en ambos bordes de los ríos consistía de árboles y arbustos en una franja entre 5 y $10 \mathrm{~m}$ de ancho. La vegetación arbórea consistía en un solo dosel, con una altura entre 6 y $14 \mathrm{~m}$, caracterizada por las especies Bursera simaruba, Enterolobium cyclocarpum, Pachira acuatica, Stemmadenia donnell-smithii, Gliricidia sepium, Attalea cohune e Inga spp. Los bosques ribereños también se caracterizaron por un sotobosque complejo, dominado por especies arbustivas de las familias Melastomaceae, Piperaceae, Polypodiaceae y Poaceae.

Los cercos vivos consistieron en filas de uno o dos árboles de ancho que separan lotes de pastura, con un dosel arbóreo bien definido, con una altura entre 4 y $8 \mathrm{~m}$, en algunos casos con árboles emergentes de hasta $20 \mathrm{~m}$ (generalmente fueron individuos de $B$. simaruba). Las especies dominantes del estrato arbóreo más comunes fueron G. sepium, E. cyclocarpum y B. simaruba. El estrato arbustivo fue escaso, caracterizado por especies pioneras de las familias Melastomaceae y Piperaceae.

Las pasturas consistían en lotes sembrados de gramíneas para ganado vacuno. Variaron desde lotes de pastura sin ninguna vegetación arbórea, hasta lotes con árboles y palmas (Vochysia guatemalensis y Attalea cohune) dispersos y arbustos como Acacia angustissima. Ocasionalmente se encontraron individuos de Ceiba pentandra de entre 20 y $25 \mathrm{~m}$ de altura.

Muestreos de aves: Para determinar las comunidades de aves presentes en los cuatro hábitats se utilizó el método de puntos de conteo (Ralph et al. 1996). En cada hábitat por finca, se realizaron seis repeticiones de una serie de diez puntos de conteo separados entre sí por $100 \mathrm{~m}$. En cada punto, durante cinco minutos, se registraron todas las aves detectadas visual o auditivamente dentro y fuera de un radio de $30 \mathrm{~m}$. Los conteos se llevaron a cabo desde el amanecer (aproximadamente 5:30 h) hasta las 9:00 h, en marzo y abril de 1998 y 1999, para asegurar la presencia de especies migratorias. Cada serie de 10 puntos se realizó por dos personas, un observador y un registrador de datos. Los conteos fueron realizados por seis registradores distintos, los cuales se alternaron entre hábitats.

El bosque y bosque ribereño (alternativamente, "río") fueron muestreados en todas las fincas $(\mathrm{n}=5)$. El cerco vivo fue muestreado solamente en tres localidades, Cayo Piedra, Río Bonito e Higuerito, dado que estaba ausente en fincas Linares y San Andrés. El pastizal fue muestreado en cuatro fincas, y todos los sitios eran activamente pastoreados.

Análisis de datos: En los análisis estadísticos, excluimos los sobrevuelos y las especies aéreas (Apodidae e Hirundinidae), dada la dificultad de determinar si estaban o no utilizando el hábitat, y las especies nocturnas, a excepción de Glaucidium brasilianum, ya que vocalizó frecuentemente durante los conteos de mañana. Para evitar la sobreestimación y el doble conteo de individuos, sólo se tomaron en cuenta las aves detectadas dentro del radio de 30 metros.

Las comparaciones entre hábitats se realizaron para cuatro parámetros distintos: riqueza de especies, número de individuos, composición e índice de importancia para la conservación. Adicionalmente, estas comparaciones se hicieron para diferentes grupos de especies: todas las especies, especies residentes, especies migratorias, y especies dependientes de bosque tropical. Utilizamos la clasificación de Stotz et al. (1996) para determinar la dependencia al bosque. Este compendio de las aves del neotrópico incluye una lista de hábitats en los cuáles están presentes cada una de las especies, en orden de importancia. Las especies para las cuáles el bosque tropical siempreverde era el hábitat primario, secundario o terciario, fueron 
incluidas dentro del grupo de "especies de bosque”. Para las especies residentes, este grupo se subdividió en dos: 1) las especies especialistas de interior de bosque tropical siempreverde de tierras bajas y montano (de aquí en adelante, "residentes de interior"), eran aquellas para las cuáles este hábitat fue considerado por Stotz et al. (1996) como el hábitat primario; 2) las especies de borde de bosque tropical siempreverde (de aquí en adelante, "residentes de borde"), aquellas que fueron identificadas como de borde de bosque tropical siempreverde por dichos autores, o que no tenían al bosque tropical siempreverde como hábitat primario.

A pesar de que el esfuerzo de muestreo en cada sitio fue relativamente alto (seis repeticiones por sitio), consideramos que subestimamos el número de especies detectadas, dado el número de especies conocidas para la región del estudio (Eisermann 2001, Cerezo et al. 2005). Por lo tanto, estimamos el número máximo de especies mediante el estimador Jackknife 2, para todos los sitios y para cada grupo de especies evaluado (residentes de interior y borde de bosque, migratorias de bosque). El estimador Jackknife 2 es considerado de alta exactitud y buena precisión, con respecto a otros estimadores del número de especies (Walther y Moore 2005).

La variación en la habilidad del observador, el hábitat y las condiciones ambientales pueden influenciar la probabilidad de detección de las aves en los puntos de conteo (Farnsworth et al. 2002, Farnsworth et al. 2005, McCallum 2005). En el presente estudio esperamos que la probabilidad de detección fuera mayor en los hábitats más abiertos, particularmente en el cerco vivo y el pastizal, y esperamos una subestimación del número de individuos, particularmente en el bosque.

Para evaluar la diferencia en la composición de especies de los cuatro hábitats, utilizamos el análisis de correspondencia sin tendencia (Hill y Gauch 1980). El método de ordenación rectificado o análisis de correspondencia sin tendencia (DCA, por las siglas del nombre en inglés) es un método de ordenamiento que, como el análisis de componentes principales, consiste en la representación de objetos (sitios, estaciones, muestreos) como puntos a lo largo de uno o varios ejes (Legendre y Legendre 1998). Los análisis de correspondencia y análisis de correspondencia rectificado pueden considerarse como un análisis del autovalor de una tabla de contingencia multivariada (Kenkel et al. 2002), en este caso, una matriz de especies (filas) por sitios (columnas). Los elementos de esta matriz fueron las abundancias relativas de las especies (la proporción de individuos de cada especie de todos los individuos detectados en el sitio). A diferencia del análisis de componentes principales y el análisis de correspondencia, el DCA rectifica la aglomeración de datos generada por el efecto de arco (Legendre y Legendre 1998), dándole una nueva escala a los ejes. Los valores en los ejes proveen un índice de la composición de especies para los sitios y un valor de afinidad a los sitios entre especies (Lieberman et al. 1996). Para evaluar las diferencias en la composición de las comunidades de cada uno de los sitios, utilizamos los valores del eje 1 del DCA correspondientes a los sitios, para poner a prueba la hipótesis nula de no diferencia en la composición de especies entre hábitats. Se realizó un análisis de correspondencia sin tendencia para cada grupo de especies.

Utilizando los valores del eje 1 del DCA para todas las especies y sitios, se construyó un listado ecológico que demuestra el cambio en la composición de especies (filas) y su afiliación a los diferentes hábitats y sitios (columnas), de forma similar a una lista fitosociológica para plantas (Lieberman et al. 1996, Perelman et al. 2002).

Para definir la importancia relativa $(I)$ de los cuatro hábitats para la conservación de las especies de bosque, utilizamos el índice desarrollado por Petit y Petit (2003), que consiste en un puntaje acumulativo basado en la fórmula:

$$
I_{j}=\sum_{i=1}^{n} v_{i} p_{i j}
$$

donde $v_{i}$ es un puntaje de vulnerabilidad para cada especie el cual representa el atributo "sensitividad a la perturbación” (baja $=1$, media $=$ 
2, alta $=3$ ) presentado por Stotz et al. (1996), $n$ es el número de especies y $p_{i j}$ es la "preferencia" relativa, calculada como la proporción del número total de individuos de la especie $i$ detectado en el habitat $j$. Por lo tanto, para cada especie, $p_{i j}$ varía entre 0 y 1 dentro del hábitat y suma 1 entre todos los hábitats.

Para todas las comparaciones estadísticas entre hábitats y diferentes grupos de especies de la riqueza (número de especies), abundancia (número de individuos), composición e índice de importancia de conservación, se utilizó el análisis de varianza de una vía (ANOVA, g.l. = 13). Al rechazar la hipótesis nula de no diferencia entre hábitats, se realizó la prueba post-hoc de comparaciones múltiples de Duncan (1951), para determinar diferencias entre pares de hábitats. Para todas las pruebas estadísticas, se utilizó un nivel de significación de $.05(\alpha=.05)$. Los datos fueron sometidos a una transformación por rangos previo al análisis de varianza, para cumplir con los supuestos de normalidad y homogeneidad de varianzas (Potvin y Roff 1993). Todos los resultados reportados en la Fig. 1 corresponden al promedio del número de especies, individuos, valores de composición e índice de importancia, en una serie de 10 puntos de conteo por hábitat, detectados dentro de un radio de 30 metros.

\section{RESULTADOS}

Un total de 248 especies fueron detectadas en el estudio, de las cuáles 180 fueron residentes y 68 migratorias (residentes invernales y transeúntes). De éstas, 210 fueron detectadas dentro del radio de $30 \mathrm{~m}$, y después de excluir los sobrevuelos, especies aéreas y nocturnas, todos los análisis se realizaron con 194 especies. De éstas, 148 fueron residentes y 46 migratorias (76.3 y $23.7 \%$, respectivamente).

El cuadro 1 presenta la riqueza de aves por hábitat y cuántas fueron detectadas en diferentes combinaciones de hábitats. Cuarenta y tres (43) de 194 especies ( $22.1 \%$ del total, cuadro 1$)$ fueron detectadas solamente en bosque, de las cuales la mayoría (42) fueron residentes. En los hábitats más abiertos (cerco vivo y pastizal) se registraron 60 especies que no fueron detectadas en bosque (30.9\% del total). En el bosque ribereño se registraron 20 especies exclusivas a él (13.6\%). El cerco vivo y pastizal estuvieron dominados por especies generalistas, las cuales se encontraron en por lo menos tres hábitats.

Riqueza de especies: El número estimado de especies fue mayor en bosque considerando todas las especies y las especies residentes (Fig. 1A), pero sólo fue estadísticamente diferente de río y cerco vivo para las residentes (Duncan $\mathrm{p}<$ $.05)$. La riqueza de especies migratorias fue significativamente mayor en río y cerco vivo que en bosque y pastizal (Fig. 1A, Duncan $\mathrm{p}<.05$ ). La riqueza de especies migratorias de bosque fue aproximadamente igual a la del pastizal (19 contra 20 especies, respectivamente), y no difirieron estadísticamente (Duncan, $\mathrm{p}>.05$ ).

Con respecto a las especies típicas de bosque (Fig. 1B), no hubo diferencia significativa en el número de especies de borde entre los 4 hábitats (ANOVA, $\mathrm{p}>.05$ ), mientras que sí hubo diferencias significativas para las especies de interior de bosque (ANOVA, $\mathrm{p}<.05$ ): el bosque tuvo casi tres veces la cantidad de especies de interior que el río (56 contra 21, respectivamente), y casi 10 veces la cantidad de especies de interior que se estimó para pastizal (6 especies). Con respecto a las migratorias de bosque, el río tuvo la mayor riqueza estimada (20 especies), seguida por el bosque (16), cerco vivo (12) y pastizal (7 especies).

CUADRO 1

Número de especies detectadas por hábitat, y número de especies que utilizaban distintas combinaciones de hábitats

TABLE 1

Species richness by habitat, and number of species that used different combinations of habitats

\begin{tabular}{lccccc}
\hline HABITAT & \multicolumn{9}{c}{ Número de hábitats utilizados } & TOTAL \\
\cline { 1 - 4 } & 1 & 2 & 3 & 4 & \\
Bosque & 43 & 37 & 23 & 31 & 134 \\
Río & 20 & 38 & 44 & 31 & 133 \\
Cerco vivo & 3 & 18 & 37 & 31 & 89 \\
Pastizal & 3 & 5 & 31 & 31 & 70
\end{tabular}



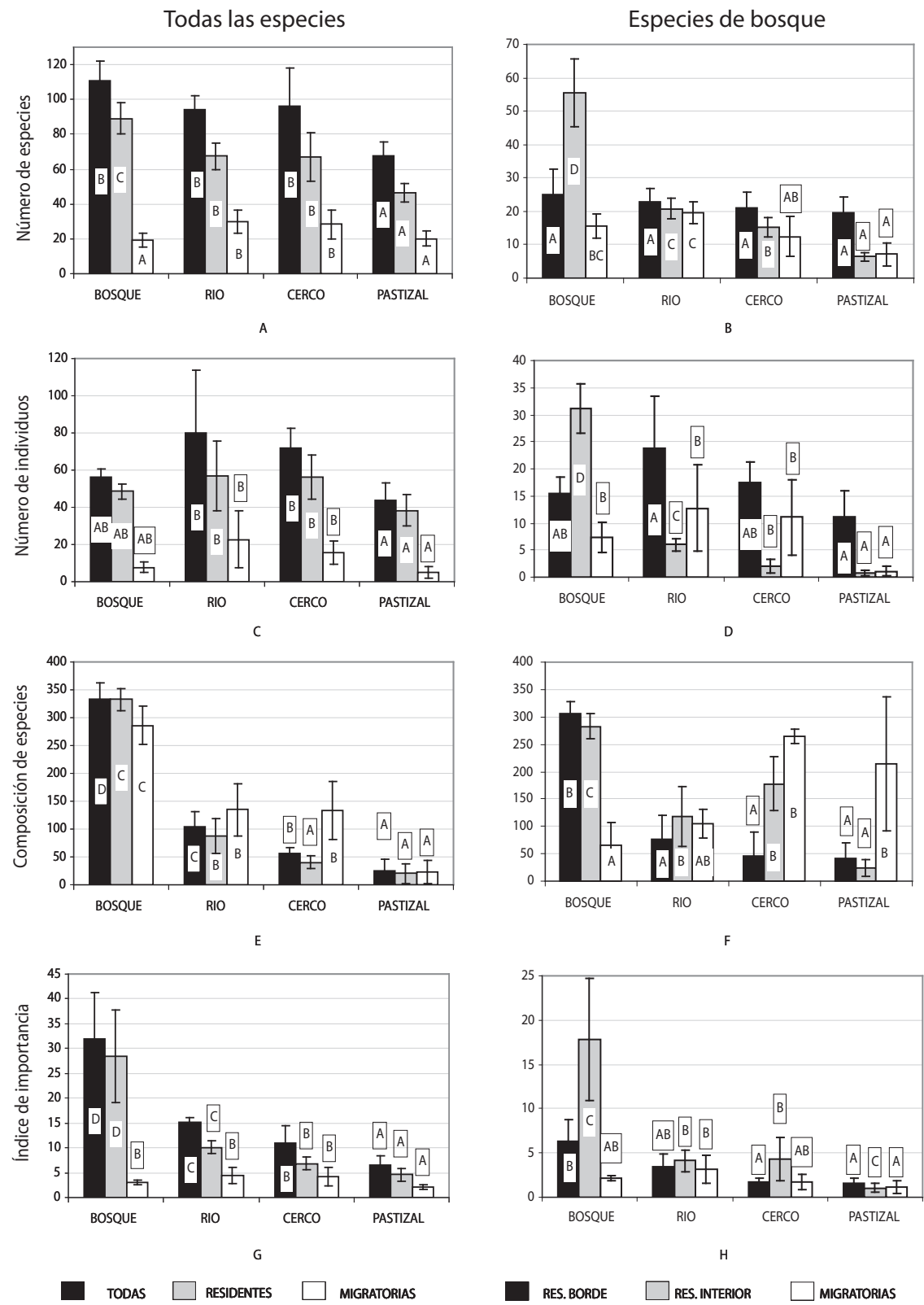

RES. BORDE $\square$ RES. INTERIOR $\square$ MIGRATORIAS

Fig. 1. Comparaciones entre hábitats en el número de especies (A y B) e individuos (C y D), composición de especies (E y

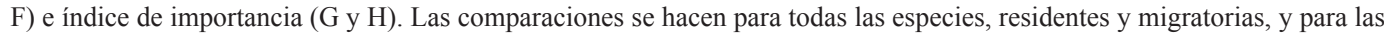
especies residentes de interior y borde de bosque y las migratorias de bosque. Los valores promedio por hábitat y serie de 10 puntos de conteo se presentan con su intervalo de confianza del 95\%; las letras mayúsculas distintas indican diferencias estadísticamente significativas entre hábitats.

Fig. 1. Comparisons between habitats in the number of species (A and B) and individuals (C and D), species composition $(\mathrm{E}$ and $\mathrm{F})$, and conservation importance index $(\mathrm{G}$ and $\mathrm{H})$. Comparisons are made for all species, residents and migrants, forest edge-dependent and forest interior-dependent residents, and forest-dependent migrants. Average values by habitat and 10 point-count series are shown with $95 \%$ confidence interval; different capital letters indicate statistically significant differences between habitats. 


\begin{abstract}
Abundancia: El número de individuos fue mayor en río y cerco vivo, para todas las especies, las especies residentes y migratorias, aunque estas diferencias no fueron estadísticamente significativas entre río, cerco vivo y bosque (Fig. 1C, Duncan $\mathrm{p}>$.05). El bosque no tuvo diferencias con ningún hábitat en estos tres parámetros (Fig. 1C, Duncan $\mathrm{p}>.05$ ), el río y cerco vivo tuvieron números de individuos migratorios significativamente distintos y mayores que el pastizal (Duncan $\mathrm{p}<.05$ ).

Con respecto al número de individuos de especies de bosque (Fig. 1D), no hubo diferencias significativas entre hábitats para el número de individuos residentes de borde (ANOVA, $\mathrm{p}>.05$ ), pero sí las hubo para las residentes de interior, donde el número de individuos fue 5 veces mayor en bosque que en río (ANOVA $p<.05,31$ en bosque contra 6 en río). Las especies migratorias de bosque fueron más abundantes en río y cerco vivo, seguidos por bosque y pastizal. Como en el caso de la riqueza de especies migratorias de bosque, no hubo diferencias significativas en el número de individuos migratorios de bosque entre río, cerco vivo y bosque (Duncan $\mathrm{p}>$.05), y bosque tampoco fue distinto del pastizal (Duncan $\mathrm{p}>$ .05 , Fig. 1D).
\end{abstract}

Composición de especies: Considerando todas las especies, la distancia o diferencia en la composición aumentó de forma gradual desde bosque hacia río, cerco vivo y pastizal (Fig. 1E) y las comparaciones de pares fueron todas significativas (Duncan $\mathrm{p}<.05$ ). Este patrón se repite para las residentes, aunque con mayores distancias relativas entre el bosque y los otros tres hábitats, y sin diferencias significativas entre cerco vivo y pastizal (Duncan $\mathrm{p}>$.05). El río y cerco vivo no mostraron diferencias estadísticamente significativas en la composición de especies migratorias, pero hubo diferencias significativas entre el bosque $\mathrm{y}$ estos dos hábitats (Duncan $\mathrm{p}<0.05$ ). El pastizal tuvo diferencias significativas con los tres hábitats en la composición de especies migratorias (Duncan $\mathrm{p}<.05$ ).

Con respecto a la composición de residentes de borde, el bosque fue diferente de los otros hábitats, pero estos no fueron diferentes entre sí (Duncan $\mathrm{p}<.05$, Fig. 1F). Para las especies de interior, existieron diferencias entre el bosque y los otros tres hábitats, el cerco vivo y río no se diferenciaron entre sí, pero fueron estadísticamente distintos del pastizal (Duncan $\mathrm{p}<.05)$. La composición de migratorias de bosque no fue significativamente diferente entre el bosque y río (Duncan $\mathrm{p}>.05$ ), y el río no fue distinto del cerco y pastizal (Duncan $\mathrm{p}>$ $.05)$. Sin embargo, el bosque sí fue distinto de cerco vivo y pastizal (Duncan $\mathrm{p}<.05$ ).

Índice de importancia: El índice de importancia del bosque en todas las comparaciones fue mayor que el de los otros hábitats (Fig. $1 \mathrm{G} \mathrm{y} 1 \mathrm{H}$ ), por una mayor abundancia en el bosque de las especies consideradas relativamente vulnerables a la perturbación (Stotz et al. 1996, Petit y Petit 2003; ver métodos). Estas diferencias fueron estadísticamente significativas entre el bosque y los otros tres hábitats considerando todas las especies, las residentes y migratorias (ANOVA, $\mathrm{p}<.05$ para todas las comparaciones; Fig. 1G).

Mientras que el río, cerco vivo y pastizal tuvieron muy poca importancia para las residentes de interior, el río tuvo un índice de importancia promedio comparable con bosque para las residentes de borde (Duncan $\mathrm{p}>.05$, Fig. 1H). El río fue el más importante para las migratorias de bosque, seguido por bosque y cerco vivo. El pastizal tuvo valores de importancia casi nulos para las residentes de interior y migratorias de bosque, y menor pero no estadísticamente diferente que los de cerco vivo y río para las residentes de borde.

Listado ecológico: En el listado ecológico (apéndice 1), las especies en la parte superior estuvieron más afiliadas al bosque, según los valores del eje 1 del DCA para todas las especies. La mayoría de las especies designadas como residentes de interior se encontraron en la parte superior de la tabla. Estas fueron seguidas por las residentes de borde y las migratorias de bosque (que en esta tabla sí están clasificadas como de borde o interior), y por último las 
especies que no son típicas de hábitats boscosos. La tabla ecológica también indica que las especies con mayor afiliación a bosque también tuvieron menor amplitud de hábitat, mientras que aquellas que se encontraron en los hábitats abiertos por lo general utilizaban tres o cuatro hábitats, es decir, fueron generalistas. Por ejemplo, de las 134 especies de bosque, 43 se detectaron solamente en este hábitat, mientras que de las 133 detectadas en río, 20 se detectaron solamente en él. La mayoría de especies detectadas en el cerco vivo y el pastizal fueron especies generalistas (ver también el cuadro 1).

Los valores del eje 1 del DCA para las especies y los sitios se corresponden: por ejemplo, las especies con valores del eje 1 cercanos al valor promedio de los sitios de bosque $( \pm$ 1 desviación estándar) son más abundantes en este hábitat. Siete especies detectadas en bosque tuvieron valores superiores al valor máximo de este intervalo (ver apéndice, valor del eje 1 para estas especies igual a 494). Estas fueron raras y solo fueron detectadas en un sitio de bosque (en finca Higuerito). Un total de 65 especies (33.5\% del total) tenían valores dentro del intervalo de una desviación estándar por arriba y debajo de la media del bosque. De éstas, 27 fueron detectadas en río y 7 en cerco vivo, con abundancias en promedio seis veces menores que en bosque. En el caso del río, 44 especies estuvieron dentro del intervalo mencionado correspondiente a este hábitat, de las cuáles 19 sólo fueron detectadas en río, y 21 fueron detectadas en tres o cuatro hábitats. En el cerco vivo, 22 especies fueron detectadas en el intervalo correspondiente a este hábitat, de las cuáles tres sólo fueron detectadas en este hábitat, y 16 en por lo menos tres. Sólo cuatro especies tuvieron valores en el intervalo correspondiente a pastizal, de las cuáles dos fueron exclusivas a este.

\section{DISCUSIÓN}

Una porción significativa de la riqueza total de aves del área de estudio está representada en los hábitats modificados, particularmente en el bosque ribereño y cerco vivo. Al considerar todas las especies, tanto el río como el cerco vivo sustentan una riqueza de especies y abundancia comparables con la de bosque. Los hábitats abiertos son incluso más diversos y sustentan mayores números de especies e individuos migratorios que el bosque. Sin embargo, debe tomarse en cuenta que probablemente se haya subestimado el número de individuos en bosque, por probabilidades de detección menores en éste. Al considerar las especies de bosque, y en particular las residentes de bosque, se detectan mayores diferencias entre el bosque y los hábitats abiertos, particularmente entre el bosque y el río y cerco vivo.

Los hábitats modificados también sustentan altos números de especies e individuos de residentes de borde de bosque. Sin embargo, la composición de residentes de borde del río y cerco es significativamente diferente a la del bosque, y consiste principalmente de especies generalistas, es decir, especies que fueron detectadas en los cuatro hábitats y con abundancia muy baja en bosque. Por otro lado, las residentes de borde que tienen una alta abundancia en bosque por lo general tienen abundancias muy bajas en el río y cerco vivo, y están prácticamente ausentes del pastizal (ver apéndice). Por ejemplo, las cuatro especies de borde más abundantes, Amazona autumnalis, Myiozetetes similis, Dives dives y Turdus grayi, fueron detectadas en los cuatro hábitats, y en abundancias mucho más altas en los hábitats modificados que en bosque. Estas especies también son consideradas generalistas, tanto por Stotz et al. (1996), quienes fueron nuestra referencia para designarlas al grupo de especies de borde, así como en otros estudios (Estrada et al. 1997, Hughes et al. 2002, Rotenberg 2007). La quinta especie de borde más abundante, Oncostoma cinereigulare, se encontró solamente en bosque y río, pero fue aproximadamente 55 veces más abundante en bosque que en río (apéndice 1). En contraste, de las 11 residentes de borde detectadas solamente en un hábitat, seis de éstas se encontraron solamente en bosque. Las otras cinco especies sólo se detectaron en río, y en abundancias muy bajas (promedio = $0.11 \pm 0.07$ individuos/10 puntos). De todas las 
especies de este grupo que tuvieron su mayor abundancia en bosque, sólo dos, Thryothorus maculipectus y Polioptila plumbea, tuvieron abundancias comparables con las del bosque (ver apéndice 1). De aquí que concluimos que los hábitats modificados probablemente tengan algún valor para un subconjunto de las especies designadas de borde, pero éstas resultaron ser especies bastante generalistas en todo su ámbito de distribución y aparentemente dependen más de los hábitats modificados que del bosque tropical (Stiles y Skutch 1989, Howell y Webb 1995, Stotz et al.1996). Las diferencias entre hábitats se reflejan claramente al evaluar la composición de especies de estos utilizando el análisis de correspondencia rectificado.

Los números de especies e individuos de las residentes de interior fueron bajos en el río y cerco vivo en comparación con el bosque. Las especies de interior que fueron detectadas en estos hábitats, se encontraron en abundancias muy bajas (Fig. 1D). Por ejemplo, la abundancia promedio de 28 residentes de interior que fueron detectadas en por lo menos un hábitat modificado varió entre $0.21,0.24$ y 0.44 individuos para pastizal, cerco vivo y río, respectivamente, mientras que en bosque este promedio fue de 1.62 individuos. De éstas, 25 fueron detectadas en río, 13 en cerco vivo y siete en pastizal. En consecuencia, la composición de especies residentes de interior de los hábitats modificados también fue significativamente diferente de la de bosque (Fig. 1F). Consideramos que muy pocas de estas especies puedan subsistir en los hábitats modificados en ausencia de bosque. Algunas especies residentes de borde fueron detectadas solamente en río o cerco vivo, resultado contraintuitivo. Sin embargo, esto se explica por el hecho de que estas especies son de por sí raras en el área de estudio (pero relativamente comunes en la región) a las elevaciones muestreadas o tienen baja detectabilidad incluso en su hábitat primario. Estas son Psarocolius wagleri, Florisuga mellivora, Heliothryx barroti (detectadas en río), y Cotinga amabilis (detectada en cerco vivo).

El pastizal resulta ser un hábitat relativamente rico en especies como conjunto, pero con muy poco valor para las especies de bosque. En términos de composición de especies, su comunidad de aves es un subconjunto de aquellas del cerco vivo, por lo que probablemente no tenga mucho valor en la ausencia del cerco (apéndice 1). Como en otros estudios (Estrada et al. 1997, Daily et al. 2001, Petit y Petit 2003, Rotenberg 2007), el valor del pastizal para las residentes de bosque es prácticamente nulo.

El caso de las especies migratorias es distinto al de las residentes de bosque. El bosque ribereño y cerco vivo parecen ser hábitats muy importantes para éstas, e incluso más importantes que el bosque, según los resultados. La mayor capacidad de utilizar hábitats distintos a bosque puede explicarse por menores requerimientos de hábitat durante el invierno neártico (Yahner 1985, en Villaseñor y Hutto 1995). Asimismo, otros estudios han encontrado que las especies migratorias dan amplio uso a hábitats modificados (Kricher y Davis 1992, Saab y Petit 1992, Greenberg et al. 1992, Hutto 1992, Robbins et al. 1992, Villaseñor y Hutto 1995). Aunque la composición de especies es muy distinta entre el bosque y la combinación ríocerco al considerar todas las especies migratorias (Fig. 1E), la composición de especies no es distinta entre el bosque y el río al considerar las migratorias que típicamente se encuentran en hábitats boscosos (Fig. 1F). Por otro lado, el cerco vivo tiene las comunidades de aves migratorias de bosque más singular. Por lo tanto, concluimos que los hábitats modificados, particularmente el río y el cerco vivo, son muy importantes para la subsistencia de especies migratorias en paisajes ganaderos.

El índice de importancia (Petit y Petit 2003) sólo es aplicable para establecer el valor de conservación de un hábitat modificado con respecto al hábitat original de la localidad de estudio. En nuestro caso, este contexto se basa en la capacidad de un hábitat de sustentar proporciones importantes de individuos de especies típicas de bosque tropical bajo, el hábitat original de nuestra área de estudio, que es de interés por estar siendo reemplazado de manera extensiva por aprovechamientos ganaderos y cultivos. Por lo tanto, calcular este índice para 
el bosque sólo tiene sentido como base comparativa para los otros hábitats. De acuerdo a los resultados, y en el caso de todas las especies, los tres hábitats modificados estudiados tienen valores muy inferiores y significativamente diferentes al de bosque, y considerando las especies de interior de bosque, los tres tienen valores casi insignificativos en comparación con el bosque (Fig. 1H). Incluso para las especies de borde, sólo el río no es significativamente diferente del bosque, aunque con aproximadamente la mitad del valor de bosque. Esto claramente se explica al evaluar la composición de especies de borde de ambos hábitats, la cual es muy diferente, y por el hecho de que las especies de borde abundantes en el bosque tienen una sensitividad media a la perturbación, y aquellas encontradas en el río tiene baja sensitividad (Stotz et al. 1996).

De la discusión previa surgen dos conclusiones fundamentales de nuestro estudio: la importancia de un hábitat alternativo para la conservación de especies debe evaluarse principalmente, 1) en función de las especies del hábitat primario, y 2) en función de la composición de especies, y no exclusivamente por el número o abundancia de todas las especies. De utilizarse solamente como criterios de evaluación el número de especies o la abundancia de todas las especies sin tomar en cuenta las diferencias en composición y sin discriminar si eran especies dependientes o no de bosque tropical, se hubiera concluido erróneamente que tanto el río como el cerco vivo son hábitats importantes para las especies de bosque. Este punto es importante, dado que por diferencias en la detectabilidad principalmente entre bosque y los otros hábitats, probablemente se esté subestimando el número de individuos residentes, y el número de especies e individuos migratorios en el bosque, que vocalizan con menor frecuencia o tienen vocalizaciones difíciles de distinguir.

Incluso en el caso de las residentes de borde, los hábitats modificados parecen tener poco valor: el índice tiene un valor muy bajo para el cerco vivo, y el de río es comparable con el de bosque pero aún así bajo. En conclusión, estos dos hábitats, a pesar de sustentar altos números de especies e individuos de aves, parecen tener poco valor de conservación para las residentes de bosque, conclusión compartida por Petit y Petit (2003) para otros hábitats modificados. Por lo tanto, aunque los hábitats modificados tienen un valor importante de conservación para la avifauna local en conjunto, y en particular para las aves migratorias, es claro que la conservación de grandes extensiones de hábitat nativo debe seguir siendo la prioridad de conservación en esta región, y probablemente en cualquier región tropical. Aunque una pequeña proporción de la biota típica del bosque tropical puede tener la capacidad de utilizar e incluso persistir en hábitats modificados o creados por el hombre (ver Sekercioglu et al. 2007), la mayoría de ésta probablemente estará condenada a desaparecer bajo escenarios de una eliminación y reemplazo extensivo de bosques tropicales por otros hábitats.

\section{AGRADECIMIENTOS}

Queremos agradecer los comentarios y sugerencias de dos revisores anónimos de manuscrito. También agradecemos a José Miguel Ponciano, Ingrid Arias, y Cory Cline por su asistencia en el trabajo de campo. Por último, debemos reconocer a la Fundación de Pesca y Vida Silvestre de E.E.U.U. (National Fish and Wildlife Foundation), The Nature Conservancy-Tennessee y FUNDAECO por la asistencia técnica, logística y financiera del estudio.

\section{RESUMEN}

A medida que las áreas de vegetación natural en los neotrópicos estén siendo reducidas a remanentes aislados, la importancia de la vegetación secundaria para la conservación de especies aumentará. Por lo tanto, debe determinarse el valor de los hábitats modificados o creados por el hombre para la conservación de especies dependientes de hábitats originales. En este estudio, evaluamos el valor para la conservación de tres hábitats asociados a la ganadería (bosque ribereño, cerco vivo y pastizal) para las aves típicas de bosque tropical en la región caribeña de Guatemala. Estudiamos las comunidades de aves de cinco sitios de 
bosque tropical bajo y bosque ribereño (río), tres sitios de cerco vivo y cuatro de pastizales, en cinco fincas ganaderas de la región, y comparamos los cuatro hábitats en función de los siguientes parámetros: número de especies, número de individuos, composición de especies, e índice de importancia. Una serie de 10 puntos de conteo se evaluó (seis repeticiones de cada serie) en cada hábitat, durante abril y mayo de 1998 y 1999. Aunque el número de especies fue similar en bosque, río y cerco vivo (111, 96 y 94 especies, respectivamente), y el río y cerco vivo superaron al bosque en el número de individuos ( 80 y 72 , comparado a 56 individuos), el río y cerco vivo tuvieron números de especies e individuos de residentes de interior de bosque significativamente menores a los del bosque $(56,21$ y 15 especies y 61, 19 y 8 individuos en bosque, río y cerco, respectivamente). El río y cerco vivo no se diferenciaron en el número de especies e individuos residentes de borde de bosque, pero la composición de las comunidades de este grupo de especies fue significativamente distinta entre estos dos hábitats y el bosque. Las especies de borde que fueron relativamente abundantes en bosque tuvieron abundancias muy bajas en los hábitats modificados, y aquellas que fueron abundantes en río y cerco vivo estaban prácticamente ausentes en el bosque tropical y fueron generalistas. Con respecto a las especies migratorias, tanto el río como el cerco vivo resultaron ser muy importantes para éstas, y el río incluso fue más importante que el bosque tropical. Concluimos que estos hábitats modificados tienen muy poco valor de conservación para las especies residentes típicas de bosque tropical, pero resultan ser muy importantes para las especies migratorias, particularmente para aquellas con hábitos arbóreos. Destacamos la importancia de evaluar la importancia de de los hábitas modificados particularmente en función de la composición de especies, y en función de las especies para las cuáles se desea evaluar la importancia de los hábitats modificados, en nuestro caso, para las especies típicas de bosque tropical.

Palabras clave: uso de hábitat, aves, bosque tropical, bosque ribereño, cerco vivo, pastizal, importancia de conservación.

\section{REFERENCIAS}

Cerezo, A., M. Ramírez \& H. Enriquez. 2005. Listado de aves de Cerro San Gil. Fundación para el Ecodesarrollo y la Conservación (FUNDAECO). Ciudad de Guatemala, Guatemala, Guatemala.

Daily, G.C., P.R. Ehrlich \& A. Sánchez-Azofeifa. 2001. Countryside biogeography: use of human-dominated habitats by the avifauna of southern Costa Rica. Ecol Appl. 11: 1-13.

Duncan, D.B. 1951. Multiple range and multiple F tests. Biometrics 11: 1-42.
Eisermann, K. 2001. Caracterización de la avifauna de Punta Manabique, Izabal. Fundación Mario Dary. Informe inédito. Ciudad de Guatemala, Guatemala, Guatemala.

Estrada, A., R. Coates-Estrada \& D.A. Meritt, Jr. 1997. Anthropogenic landscape changes and avian diversity at Los Tuxtlas, Mexico. Biodivers. Conserv. 6: 19-43.

Farnsworth, G.L., K.H. Pollock, J.D. Nichols, T.R. Simons, J.E. Hines \& J.R. Sauer. 2002. A removal model for estimating detection probabilities from point-count surveys. Auk 119: 414-425.

Farnsworth, G.L., J.D. Nichols, J.R. Sauer, S.G. Fancy, K.H. Pollock, S.A. Shriner \& T.R. Simons. 2005. Statistical approaches to the analysis fo point count data: a little extra information can go a long way, p. 736-743. In C. J. Ralph \& T. D. Rich (eds.). Bird conservation implementation and integration in the Americas: Proceedings of the Third International Partners in Flight Conference, General Technical Report PSW-GTR-191. Albany, California, EEUU.

FAO. 1999. State of the world's forests. Food and Agriculture Organization of the United Nations, Rome, Rome, Italy.

FAO. 2005. Global Forest Resources Assessment 2005. FAO Forestry Paper No. 147. Food and Agriculture Organization of the United Nations, Rome, Rome, Italy.

Greenberg, R. 1992. Forest migrants in non-forest habitats on the Yucatan Peninsula, p. 273-286. In J.M. Hagan III \& D.W. Johnston (eds.). Ecology and conservation of Neotropical migrant landbirds. Smithsonian, Washington D.C., EEUU.

Harris, G.M. \& S.L. Pimm. 2004. Bird species tolerance of secondary habitats and its effects on extinction. Conser. Biol. 18: 1607-1616.

Harvey, C.A., A. Medina, D.M. Sánchez, S. Vilcehz, B. Hernández, J.C. Saenz, J.M. Maes, F. Casanoves \& F.L. Sinclair. 2006. Patterns of animal diversity in different forms of tree cover in agricultural landscapes. Ecol. Appl. 16: 1986-1999.

Hill, M.O. \& H.G. Gauch. 1980. Detrended correspondence analysis: an improved ordination technique. Vegetatio 42: 47-58.

Holdridge, L.R. 1970. Life zone ecology. Tropical Science Center, San José, San José, Costa Rica.

Howell, S.N.G. \& S. Webb. 1995. A guide to the birds of Mexico and northern Central America. Oxford University, New York, New York, EEUU. 
Hughes, J.B., G.C. Daily \& P.R. Ehrlich. 2002. Conservation of tropical forest birds in countryside habitats. Ecol. Lett. 5: 121-129.

Hutto, R.L. 1992. Habitat distributions of migratory landbird species in western Mexico, p. 273-286. In J.M. Hagan III \& D.W. Johnston (eds.). Ecology and conservation of Neotropical Migrant Landbirds. Smithsonian, Washington D.C., EEUU.

Janzen, D.H. 1988. Tropical dry forests: the most endangered major tropical ecosystem, p. 130-137. In E.O. Wilson (ed.). Biodiversity. National Academy of Sciences, Washington D.C., EEUU.

Kaimowitz, D. 1996. Livestock and deforestation, Central America in the 1980s and 1990s: a policy perspective. Center for International Forestry Research Special Publication, Jakarta, Jabar, Indonesia.

Kenkel, N.C, D.A. Derksen, A.G. Thomas \& P.R. Watson. 2002. Multivariate analysis in weed science research. Weed Sci. 50: 281-292.

Kricher, J.C. \& W.E. Davis. 1992. Patterns of avian species richness in disturbed and undisturbed habitats in Belize, p. 273-286. In J.M. Hagan III \& D.W. Johnston (eds.). Ecology and conservation of Neotropical migrant Landbirds. Smithsonian, Washington D.C., EEUU.

Legendre, P. \& L. Legendre. 1998. Numerical ecology. Elsevier, Amsterdam, Holland.

Lieberman, D., M. Lieberman, R. Peralta \& G.S. Hartshorn. 1996. Tropical forest structure and composition on the large-scale altitudinal gradient in Costa Rica. J. Ecol. 84: 137-152.

Ludwig, J. \& J. Reynolds. 1988. Statistical ecology: a primer on methods and computing. John Wiley \& Sons, New York, New York, EEUU.

McCallum, D.A. 2005. A conceptual guide to detection probability for point counts and other count-based survey methods, p. 754-761. In C. J. Ralph \& T. D. Rich (eds.). Bird conservation implementation and integration in the Americas: Proceedings of the Third International Partners in Flight Conference, General Technical Report PSW-GTR-191. Albany, California, EEUU.

McCune, B. \& M.J. Mefford. 1999. PC-Ord for Windows. Multivariate analysis of ecological data. Version 4.0. MjM Software, Glenenden Beach, Oregon, EEUU.
Petit, L.J., D.R. Petit, D.G. Christian \& H.D.W. Powell. 1999. Bird communities of natural and modified habitats in Panama. Ecography 22: 292-304.

Petit, L.J. \& D.R. Petit. 2003. Evaluating the importance of human-modified lands for Neotropical bird conservation. Conser. Biol. 17: 687-694.

Perelman, S., R.J.C. León \& M. Oesterheld. 2001. Crossscale vegetation patterns of flooding pampa grasslands. J. Ecol. 89: 562-577.

Pimm, S.L. \& P. Raven. 2000. Extinction by numbers. Nature 403: 843-845.

Pimm, S.L., G.J. Russell, J.L. Gittleman \& T.M. Brooks. 1995. The future of biodiversity. Science 269: $347-$ 350 .

Potvin, C. \& D. Roff. 1993. Distribution-free and robust statistical methods: viable alternatives to paremetric statistics? Ecology 74: 1617-1628.

Ralph, C.J., G.R. Geupel, P. Pyle, T.E. Martin, D. DeSante \& B. Milá. 1996. Manual de métodos de campo para el monitoreo de aves terrestres. General Technical Report PSW-GTR-159. Albany, California, EEUU.

Robbins, C.S., B.A. Dowell, D.K. Dawson, J.A. Colón, R. Estrada, A. Sutton, R. Sutton \& D. Weyer. 1992. Comparison of Neotropical migrant landbird populations wintering in tropical forest, isolated forest fragments, and agricultural habitats, p. 207-220. In J.M Hagan III \& D. Johnston (eds.). Ecology and conservation of Neotropical migrant landbirds. Smithsonian Institution, Washington D.C., EEUU.

Rotenberg, J.A. 2007. Ecological role of a tree (Gmelina arborea) plantation in Guatemala: an assessment of an alternative land use for tropical avian conservation. Auk 124: 316-330.

Saab, V. \& D.R. Petit. 1992. Impact of pasture development on winter bird communities in Belize, Central America. Condor 94: 66-71.

Sekercioglu, C.H., S.R. Loarie, F. Oviedo, P.R. Ehrlich \& G.C. Daily. 2005. Persistence of forest birds in the Costa Rican agricultural countryside. Cons. Biol. 21: 482-494.

Smith, A.L., J.S. Ortiz \& R.J. Robertson. 2001. Distribution patterns of migrant and resident birds in successional 
forests of the Yucatan Peninsula. Mexico. Biotropica 33: $153-170$.

Stiles, F.G. \& A.F. Skutch. 1989. A guide to the birds of Costa Rica. Cornell University. Ithaca, New York, EEUU.

Stotz, D.F., J.W. Fitzpatrick, T.A. Parker III \& D.K. Moskovits. 1996. Neotropical birds: ecology and conservation. University of Chicago, Chicago, Illinois, EEUU.

Thornthwaite, C.W. 1948. An approach toward a rational classification of climate. Geogr. Rev. 38: 55-94.

Vandermeer, J. \& I. Perfecto. 1997. The agroecosystem: a need for the conservation biologist's lens. Conser. Biol. 11: 591-592.

Villaseñor, J.F. \& R.L. Hutto. 1995. The importance of agricultural areas for the conservation of Neotropical migratory landbirds in western Mexico, p. 59-80. In M.H. Wilson \& S.A. Sader (eds.). Conservation of neotropical migratory birds in Mexico. Agricultural and Forest Experiment Station Miscellaneous Publication 727. Orono, Maine, EEUU.

Yahner, R.H. 1985. Effects of forest fragmentation on winter bird abundance in Central Pennsylvania. Proc. Pennsylvania Acad. Sci. 59: 114-116.

Walther, B.A. \& J.L. Moore. 2005. The concepts of bias, precision and accuracy, and their use in testing the performance of species richness estimators, with a literature review of estimator performance. Ecography 28: $815-829$

Wilcove, D.S. 1985. Nest predation and the decline of migratory songbirds. Ecology 66: 1211-1214.

\section{REFERENCIAS DE INTERNET}

INSIVUMEH. 2003. Datos meteorológicos de la estación Puerto Barrios, Izabal, Guatemala, para el período 1993-2003. Instituto Nacional de Sismología, Vulcanología, Meteorología e Hidrología, Ciudad de Guatemala, Guatemala, Guatemala (Fecha de consulta: 20 Febrero 2005, http://www.insivumeh.gob.gt.) 


\section{APENDICE 1}

Listado ecológico de aves detectadas y utilizadas en los análisis estadísticos. Para cada especie se muestra: condición (res-residente, mig-migratoria), grupo al cual fue asignada (interior o borde de bosque, no bos-típica de hábitats abiertos), número de hábitats en los que fue detectado (\#HABS), hábitat en el que tuvo su mayor abundancia ("mayor abund."), y número de individuos promedio por hábitat en una serie de 10 puntos de conteo.

\section{APPENDIX 1}

Ecological list of birds detected in our study and used in statistical comparisons between habitats. For each species, we show: status (res-resident, mig-migrant), assigned ecological group (interior, edge, non-forest), number of habitats where it was detected (\#HABS), habitat where abundance was greatest ("mayor abund"), and average number of individuals detected by habitat for a 10-point count series.

\begin{tabular}{|c|c|c|c|c|c|c|c|c|c|}
\hline ESPECIE & $\begin{array}{c}\text { CONDI- } \\
\text { CION }\end{array}$ & GRUPO & \#HABS & $\begin{array}{l}\text { Mayor } \\
\text { abund. }\end{array}$ & $\begin{array}{l}\text { DCA } \\
\text { EJE1 }\end{array}$ & $\begin{array}{l}\text { BOSQUE } \\
374 \pm 69^{*}\end{array}$ & $\begin{array}{c}\text { BOSQUE } \\
\text { RIBEREÑO } \\
199 \pm 25^{*}\end{array}$ & $\begin{array}{c}\text { CERCO } \\
\text { VIVO } \\
106 \pm 24^{*}\end{array}$ & $\begin{array}{c}\text { PASTIZAL } \\
16 \pm 54^{*}\end{array}$ \\
\hline Hylomanes momotula & res & interior & 1 & bosque & 494 & 0.07 & & & \\
\hline Electron carinatum & res & interior & 1 & bosque & 494 & 0.22 & & & \\
\hline Sclerurus guatemalensis & res & interior & 1 & bosque & 494 & 0.15 & & & \\
\hline Xiphorhynchus erythropygius & res & interior & 1 & bosque & 494 & 0.07 & & & \\
\hline Myrmotherula schisticolor & res & interior & 1 & bosque & 494 & 0.07 & & & \\
\hline Rhytipterna holerythra & res & interior & 1 & bosque & 494 & 0.07 & & & \\
\hline Turdus assimilis & res & interior & 1 & bosque & 494 & 0.36 & & & \\
\hline Lipaugus unirufus & res & interior & 1 & bosque & 458 & 1.03 & & & \\
\hline Formicarius moniliger & res & interior & 1 & bosque & 457 & 0.66 & & & \\
\hline Mionectes oleagineus & res & interior & 1 & bosque & 439 & 2.29 & & & \\
\hline Trogon violaceus & res & interior & 1 & bosque & 438 & 0.22 & & & \\
\hline Dysithamnus mentalis & res & interior & 1 & bosque & 423 & 0.52 & & & \\
\hline Arremon aurantiirostris & res & interior & 1 & bosque & 416 & 0.14 & & & \\
\hline Dendroncincla homochroa & res & interior & 1 & bosque & 413 & 0.59 & & & \\
\hline Onychorynchus coronatus & res & interior & 1 & bosque & 412 & 0.45 & & & \\
\hline Crypturellus boucardi & res & interior & 1 & bosque & 411 & 0.30 & & & \\
\hline Thalurania colombica & res & interior & 1 & bosque & 411 & 0.15 & & & \\
\hline Melanerpes pucherani & res & interior & 1 & bosque & 411 & 0.45 & & & \\
\hline Celeus castaneus & res & interior & 2 & bosque & 401 & 2.27 & 0.08 & & \\
\hline Patagioenas nigrirostris & res & interior & 3 & bosque & 390 & 2.68 & 0.11 & 0.08 & \\
\hline Habia rubica & res & interior & 1 & bosque & 389 & 0.65 & & & \\
\hline Euphonia gouldi & res & interior & 1 & bosque & 389 & 1.61 & & & \\
\hline Platyrhinchus cancrominus & res & interior & 1 & bosque & 387 & 1.90 & & & \\
\hline Campephilus guatemalensis & res & borde & 3 & bosque & 381 & 0.66 & 0.08 & 0.09 & \\
\hline Hylophilus ochraceiceps & res & interior & 1 & bosque & 381 & 0.80 & & & \\
\hline
\end{tabular}




\begin{tabular}{|c|c|c|c|c|c|c|c|c|c|}
\hline ESPECIE & $\begin{array}{c}\text { CONDI- } \\
\text { CION }\end{array}$ & GRUPO & \#HABS & $\begin{array}{l}\text { Mayor } \\
\text { abund. }\end{array}$ & $\begin{array}{l}\text { DCA } \\
\text { EJE1 }\end{array}$ & $\begin{array}{l}\text { BOSQUE } \\
374 \pm 69 *\end{array}$ & $\begin{array}{c}\text { BOSQUE } \\
\text { RIBEREÑO } \\
199 \pm 25^{*}\end{array}$ & $\begin{array}{c}\text { CERCO } \\
\text { VIVO } \\
106 \pm 24^{*}\end{array}$ & $\begin{array}{c}\text { PASTIZAL } \\
16 \pm 54^{*}\end{array}$ \\
\hline Henicorhina leucostica & res & interior & 2 & bosque & 379 & 3.38 & 0.06 & & \\
\hline Phaethornis longirostris & res & interior & 2 & bosque & 377 & 1.60 & 0.08 & & \\
\hline Dendrocincla anabatina & res & interior & 2 & bosque & 377 & 0.34 & 0.05 & & \\
\hline Trogon collaris & res & interior & 1 & bosque & 376 & 0.29 & & & \\
\hline Falco sparverius & mig & no bos & 1 & bosque & 375 & 0.06 & & & \\
\hline Leptotila verreauxi & res & no bos & 1 & bosque & 375 & 0.06 & & & \\
\hline Microrhopias quixensis & res & interior & 1 & bosque & 375 & 0.06 & & & \\
\hline Leptotila cassinni & res & borde & 3 & bosque & 374 & 2.54 & 0.17 & 0.10 & \\
\hline Habia fuscicauda & res & borde & 1 & bosque & 373 & 1.01 & & & \\
\hline Polioptila caerulea & mig & borde & 2 & bosque & 368 & 0.44 & 0.14 & & \\
\hline Momotus momota & res & interior & 1 & bosque & 367 & 1.52 & & & \\
\hline Trogon massena & res & interior & 2 & bosque & 363 & 1.24 & 0.06 & & \\
\hline Phaethornis striigularis & res & interior & 2 & bosque & 361 & 2.99 & 0.17 & & \\
\hline Oncostoma cinereigulare & res & borde & 2 & bosque & 359 & 7.74 & 0.14 & & \\
\hline Amazona farinosa & res & interior & 1 & bosque & 358 & 0.56 & & & \\
\hline Myopagis viridicata & res & interior & 1 & bosque & 357 & 1.02 & & & \\
\hline Manacus candei & res & borde & 2 & bosque & 356 & 4.31 & 0.08 & & \\
\hline Pipra mentalis & res & interior & 2 & bosque & 356 & 2.12 & 0.67 & & \\
\hline Hylophilus decurtatus & res & interior & 2 & bosque & 351 & 6.98 & 0.36 & & \\
\hline Ramphocaenus melanurus & res & borde & 2 & bosque & 350 & 2.36 & & 0.10 & \\
\hline Ortalis vetula & res & borde & 1 & bosque & 348 & 0.34 & & & \\
\hline Hylocichla mustelina & $\operatorname{mig}$ & interior & 2 & bosque & 348 & 1.18 & 0.05 & & \\
\hline Cyanocompsa cyanoides & res & interior & 2 & bosque & 340 & 0.70 & & 0.09 & \\
\hline Pteroglossus torquatus & res & interior & 2 & bosque & 338 & 1.06 & 0.11 & & \\
\hline Claravis pretiosa & res & borde & 1 & bosque & 337 & 0.58 & & & \\
\hline Columba speciosa & res & interior & 1 & bosque & 336 & 0.07 & & & \\
\hline Galbula ruficauda & res & borde & 1 & bosque & 336 & 0.14 & & & \\
\hline Terenotriccus erythrurus & res & interior & 1 & bosque & 336 & 0.07 & & & \\
\hline Myiobius sulphureipygius & res & interior & 1 & bosque & 336 & 0.14 & & & \\
\hline Pachyramphus aglaiae & res & no bos & 1 & bosque & 336 & 0.07 & & & \\
\hline Amazilia candida & res & interior & 3 & bosque & 335 & 3.57 & 0.29 & 0.25 & \\
\hline Xiphorhynchus flavigaster & res & interior & 2 & bosque & 335 & 1.20 & 0.24 & & \\
\hline Tinamus major & res & interior & 1 & bosque & 334 & 0.90 & & & \\
\hline Crypturellus soui & res & borde & 1 & bosque & 334 & 0.08 & & & \\
\hline Spizaetus tyrannus & res & interior & 1 & bosque & 334 & 0.23 & & & \\
\hline Automolus ochroloaemus & res & interior & 1 & bosque & 334 & 0.15 & & & \\
\hline Attila spadiceus & res & interior & 2 & bosque & 334 & 1.72 & 0.30 & & \\
\hline Schiffornis turdinus & res & interior & 2 & bosque & 333 & 0.91 & 0.08 & & \\
\hline Crax rubra & res & interior & 1 & bosque & 332 & 0.08 & & & \\
\hline Leptotila rufaxilla & res & borde & 1 & bosque & 332 & 0.08 & & & \\
\hline
\end{tabular}




\begin{tabular}{|c|c|c|c|c|c|c|c|c|c|}
\hline ESPECIE & $\begin{array}{l}\text { CONDI- } \\
\text { CION }\end{array}$ & GRUPO & \#HABS & $\begin{array}{l}\text { Mayor } \\
\text { abund. }\end{array}$ & $\begin{array}{l}\text { DCA } \\
\text { EJE1 }\end{array}$ & $\begin{array}{l}\text { BOSQUE } \\
374 \pm 69^{*}\end{array}$ & $\begin{array}{c}\text { BOSQUE } \\
\text { RIBEREÑO } \\
199 \pm 25^{*}\end{array}$ & $\begin{array}{c}\text { CERCO } \\
\text { VIVO } \\
106 \pm 24^{*}\end{array}$ & $\begin{array}{c}\text { PASTIZAL } \\
16 \pm 54^{*}\end{array}$ \\
\hline Glyphorynchus spirurus & res & interior & 3 & bosque & 332 & 0.71 & 0.26 & 0.09 & \\
\hline Empidonax flaviventris & mig & interior & 2 & bosque & 329 & 1.23 & 0.22 & & \\
\hline Ornithion semiflavum & res & borde & 2 & bosque & 326 & 0.72 & 0.06 & & \\
\hline Pionopsitta haematotis & res & interior & 3 & bosque & 323 & 2.48 & 0.10 & 0.36 & \\
\hline Tolmomyias sulphurescens & res & interior & 3 & bosque & 323 & 1.81 & 0.73 & 0.19 & \\
\hline Oporornis formosus & mig & interior & 2 & bosque & 323 & 0.19 & 0.05 & & \\
\hline Thryothorus maculipectus & res & borde & 2 & bosque & 314 & 1.48 & 0.51 & & \\
\hline Thamnophilus doliatus & res & no bos & 2 & bosque & 291 & 0.19 & & 0.10 & \\
\hline Catharus ustulatus & mig & borde & 3 & bosque & 288 & 1.29 & 0.86 & 0.19 & \\
\hline Cyanocorax yncas & res & borde & 2 & río & 287 & 0.15 & 0.17 & & \\
\hline Ramphastos sulfuratus & res & interior & 4 & bosque & 282 & 1.10 & 0.50 & 0.16 & 0.16 \\
\hline Campylorhynchus zonatus & res & no bos & 2 & bosque & 279 & 0.15 & 0.10 & & \\
\hline Piculus rubiginosus & res & borde & 2 & bosque & 278 & 0.07 & 0.05 & & \\
\hline Piaya cayana & res & interior & 3 & bosque & 277 & 0.64 & 0.46 & & 0.08 \\
\hline Contopus cinereus & res & borde & 2 & bosque & 277 & 0.08 & 0.05 & & \\
\hline Caryothraustes poliogaster & res & interior & 2 & bosque & 273 & 0.37 & & 0.31 & \\
\hline Psarocolius montezuma & res & interior & 3 & bosque & 270 & 3.00 & 2.62 & & 0.56 \\
\hline Polioptila plumbea & res & borde & 4 & bosque & 268 & 0.37 & 0.08 & 0.17 & 0.22 \\
\hline Vireo olivaceus & mig & interior & 2 & río & 262 & 0.15 & 0.20 & & \\
\hline Wilsonia canadensis & mig & borde & 2 & río & 261 & 0.07 & 0.14 & & \\
\hline Dumetella carolinensis & mig & borde & 3 & bosque & 256 & 0.97 & 0.94 & & 0.17 \\
\hline Cairina moschata & res & no bos & 2 & cerco & 252 & 0.08 & & 0.09 & \\
\hline Setophaga ruticilla & mig & interior & 3 & río & 250 & 1.81 & 2.95 & & 0.08 \\
\hline Cyanerpes cyaneus & res & interior & 3 & bosque & 248 & 0.29 & 0.19 & 0.16 & \\
\hline Dendroica magnolia & mig & interior & 4 & río & 247 & 2.55 & 3.82 & 0.51 & 0.40 \\
\hline Cercromacra tyraninna & res & borde & 2 & río & 245 & 0.26 & 0.64 & & \\
\hline Myiarchus crinitus & mig & interior & 3 & bosque & 245 & 0.74 & 0.21 & 0.63 & \\
\hline Saltator atriceps & res & borde & 1 & río & 243 & & 0.08 & & \\
\hline Tityra semifasciata & res & interior & 4 & río & 229 & 1.45 & 1.48 & 1.17 & 0.14 \\
\hline Micrastur semitorquatus & res & interior & 2 & cerco & 222 & 0.07 & & 0.10 & \\
\hline Wilsonia citrina & mig & interior & 4 & bosque & 221 & 0.47 & 1.72 & 0.10 & 0.08 \\
\hline Piranga rubra & mig & no bos & 4 & río & 220 & 0.57 & 0.90 & 0.51 & 0.23 \\
\hline Dendroica pensylvanica & mig & interior & 3 & cerco & 214 & 1.54 & 1.27 & 2.58 & \\
\hline Trogon melanocephalus & res & interior & 4 & río & 212 & 0.65 & 1.10 & 0.09 & 0.36 \\
\hline Falco rufigularis & res & borde & 3 & bosque & 211 & 0.15 & & 0.10 & 0.08 \\
\hline Amazona albifrons & res & borde & 2 & pastizal & 210 & 0.44 & & & 0.52 \\
\hline Contopus virens & mig & borde & 4 & bosque & 209 & 0.36 & 0.36 & 0.32 & 0.15 \\
\hline Glaucidium brasilianum & res & no bos & 1 & río & 207 & & 0.33 & & \\
\hline Mniotilta varia & mig & interior & 3 & cerco & 206 & 0.44 & 0.63 & 0.79 & \\
\hline Herpetotheres cachinnans & res & borde & 3 & río & 202 & 0.06 & 0.15 & & 0.09 \\
\hline
\end{tabular}




\begin{tabular}{|c|c|c|c|c|c|c|c|c|c|}
\hline ESPECIE & $\begin{array}{c}\text { CONDI- } \\
\text { CION }\end{array}$ & GRUPO & \#HABS & $\begin{array}{l}\text { Mayor } \\
\text { abund. }\end{array}$ & $\begin{array}{l}\text { DCA } \\
\text { EJE1 }\end{array}$ & $\begin{array}{l}\text { BOSQUE } \\
374 \pm 69^{*}\end{array}$ & $\begin{array}{c}\text { BOSQUE } \\
\text { RIBEREÑO } \\
199 \pm 25^{*}\end{array}$ & $\begin{array}{c}\text { CERCO } \\
\text { VIVO } \\
106 \pm 24^{*}\end{array}$ & $\begin{array}{c}\text { PASTIZAL } \\
16 \pm 54^{*}\end{array}$ \\
\hline Patagioenas flavirostris & res & no bos & 2 & cerco & 199 & 0.08 & & 0.19 & \\
\hline Ictinia plumbea & res & interior & 3 & río & 196 & 0.06 & 0.37 & & 0.09 \\
\hline Geothlypis poliocephala & $\operatorname{mig}$ & no bos & 1 & río & 195 & & 0.05 & & \\
\hline Arremonops chloronotus & res & borde & 1 & río & 195 & & 0.05 & & \\
\hline Saltator maximus & res & borde & 1 & río & 195 & & 0.15 & & \\
\hline Psarocolius wagleri & res & interior & 1 & río & 195 & & 0.05 & & \\
\hline Megarynchus pitangua & res & borde & 3 & río & 194 & 0.07 & 0.39 & & 0.16 \\
\hline Wilsonia pusilla & mig & no bos & 3 & cerco & 194 & 0.14 & 0.05 & 0.31 & \\
\hline Lepidocolaptes souleyetii & res & borde & 1 & río & 192 & & 0.24 & & \\
\hline Dendrocygna autumnalis & res & no bos & 1 & río & 191 & & 0.06 & & \\
\hline Heliornis fulica & res & no bos & 1 & río & 191 & & 0.19 & & \\
\hline Florisuga mellivora & res & interior & 1 & río & 191 & & 0.06 & & \\
\hline Piranga olivacea & mig & interior & 1 & río & 191 & & 0.06 & & \\
\hline Pionus senilis & res & borde & 1 & río & 189 & & 0.05 & & \\
\hline Tapera naevia & res & no bos & 1 & río & 189 & & 0.05 & & \\
\hline Amazilia tzacatl & res & borde & 4 & cerco & 189 & 0.80 & 2.62 & 2.63 & 0.29 \\
\hline Icteria virens & mig & no bos & 1 & río & 189 & & 0.05 & & \\
\hline Tyrannus tyrannus & mig & no bos & 1 & río & 187 & & 0.36 & & \\
\hline Euphonia hirundinacea & res & borde & 3 & río & 187 & 0.08 & 1.03 & & 0.15 \\
\hline Chloroceryle amazona & res & no bos & 1 & río & 185 & & 0.16 & & \\
\hline Todirostrum cinereum & res & borde & 3 & río & 185 & & 1.59 & 0.66 & 0.17 \\
\hline Myiarchus tuberculifer & res & borde & 4 & río & 185 & 0.89 & 2.10 & 1.01 & 1.08 \\
\hline Tityra inquisitor & res & interior & 4 & río & 185 & 0.08 & 0.39 & 0.08 & 0.09 \\
\hline Cyanocorax morio & res & no bos & 4 & cerco & 184 & 1.43 & 3.30 & 3.36 & 1.19 \\
\hline Seiurus noveboracensis & mig & interior & 4 & río & 184 & 0.21 & 4.48 & 0.27 & 0.66 \\
\hline Dendroica castanea & mig & interior & 3 & cerco & 183 & 0.15 & 0.06 & 0.41 & \\
\hline Myiodynastes luteiventris & res & borde & 4 & río & 182 & 0.15 & 0.31 & 0.18 & 0.22 \\
\hline Amazona autumnalis & res & borde & 4 & río & 180 & 2.04 & 7.41 & 4.19 & 2.97 \\
\hline Heliothryx barroti & res & interior & 1 & río & 178 & & 0.05 & & \\
\hline Vireo solitarius & mig & borde & 1 & río & 178 & & 0.05 & & \\
\hline Helmitheros vermivorus & mig & interior & 1 & río & 178 & & 0.05 & & \\
\hline Seiurus motacilla & mig & interior & 1 & río & 178 & & 0.16 & & \\
\hline Spiza americana & mig & no bos & 2 & río & 174 & & 0.27 & 0.10 & \\
\hline Buteo magnirostris & res & borde & 4 & río & 171 & 0.15 & 0.52 & 0.52 & 0.29 \\
\hline Euphonia affinis & res & no bos & 3 & río & 171 & & 0.70 & 0.31 & 0.15 \\
\hline Dryocopus lineatus & res & no bos & 4 & cerco & 170 & 0.14 & 0.16 & 0.33 & 0.16 \\
\hline Aratinga nana & res & borde & 4 & río & 168 & 1.00 & 2.33 & 1.32 & 1.67 \\
\hline Aramus guarauna & res & no bos & 2 & río & 167 & & 0.42 & 0.25 & \\
\hline Geothlypis trichas & mig & no bos & 3 & río & 167 & & 1.85 & 1.22 & 0.17 \\
\hline Turdus grayi & res & borde & 4 & río & 165 & 0.29 & 4.74 & 2.06 & 0.83 \\
\hline
\end{tabular}




\begin{tabular}{|c|c|c|c|c|c|c|c|c|c|}
\hline ESPECIE & $\begin{array}{l}\text { CONDI- } \\
\text { CION }\end{array}$ & GRUPO & \#HABS & $\begin{array}{l}\text { Mayor } \\
\text { abund. }\end{array}$ & $\begin{array}{l}\text { DCA } \\
\text { EJE1 }\end{array}$ & $\begin{array}{l}\text { BOSQUE } \\
374 \pm 69 *\end{array}$ & $\begin{array}{c}\text { BOSQUE } \\
\text { RIBEREÑO } \\
199 \pm 25^{*}\end{array}$ & $\begin{array}{c}\text { CERCO } \\
\text { VIVO } \\
106 \pm 24^{*}\end{array}$ & $\begin{array}{c}\text { PASTIZAL } \\
16 \pm 54^{*}\end{array}$ \\
\hline Pheucticus ludovicianus & mig & no bos & 2 & cerco & 163 & 0.07 & & 0.29 & \\
\hline Phalacrocorax olivaceus & res & no bos & 2 & río & 162 & & 0.19 & & 0.07 \\
\hline Chloroceryle americana & res & no bos & 2 & río & 162 & & 0.84 & 0.37 & \\
\hline Melanerpes aurifrons & res & no bos & 4 & río & 162 & 1.29 & 7.17 & 5.05 & 2.42 \\
\hline Ceryle alcyon & mig & no bos & 2 & cerco & 161 & & 0.05 & 0.09 & \\
\hline Laterallus ruber & res & no bos & 3 & río & 160 & & 0.79 & 0.19 & 0.42 \\
\hline Vermivora peregrina & mig & borde & 4 & cerco & 159 & 1.42 & 2.79 & 8.81 & 0.31 \\
\hline Icterus galbula & mig & no bos & 4 & río & 159 & 0.08 & 3.37 & 1.31 & 0.95 \\
\hline Actitis macularius & mig & no bos & 2 & río & 153 & & 0.11 & 0.08 & \\
\hline Dendroica petechia & mig & no bos & 3 & río & 152 & & 7.32 & 0.70 & 2.69 \\
\hline Tigrisoma mexicanum & res & no bos & 2 & cerco & 151 & & 0.21 & 0.60 & \\
\hline Empidonax minimus & $\operatorname{mig}$ & interior & 3 & río & 151 & & 0.16 & 0.09 & 0.09 \\
\hline Pitangus sulphuratus & res & no bos & 4 & río & 148 & 0.60 & 5.07 & 3.91 & 3.16 \\
\hline Coragyps atratus & res & no bos & 4 & cerco & 147 & 0.07 & 1.69 & 1.75 & 0.47 \\
\hline Ceryle torquatus & res & no bos & 1 & río & 145 & & 0.08 & & 0.07 \\
\hline Thraupis episcopus & res & borde & 4 & río & 141 & 0.15 & 2.69 & 1.89 & 0.93 \\
\hline Thraupis abbas & res & borde & 3 & cerco & 140 & & 2.41 & 3.09 & 0.42 \\
\hline Cathartes aura & res & no bos & 4 & río & 139 & 0.08 & 0.54 & 0.21 & 0.31 \\
\hline Crotophaga sulcirostris & res & no bos & 3 & río & 131 & & 2.66 & 1.00 & 1.74 \\
\hline Pachyramphus polychopterus & res & borde & 2 & cerco & 129 & & 0.05 & 0.16 & \\
\hline Patagioenas cayennensis & res & no bos & 4 & río & 128 & & 1.00 & 0.80 & 0.53 \\
\hline Ardea alba & mig & no bos & 3 & cerco & 126 & & 0.21 & 0.61 & 0.23 \\
\hline Dendroica fusca & mig & borde & 2 & cerco & 126 & & 0.06 & 0.29 & \\
\hline Icterus spurius & mig & no bos & 3 & río & 125 & & 1.49 & 1.13 & 2.01 \\
\hline Myiozetetes similis & res & borde & 4 & río & 121 & 0.41 & 5.32 & 5.03 & 4.34 \\
\hline Tyrannus melancholicus & res & no bos & 3 & cerco & 115 & & 2.68 & 4.86 & 2.20 \\
\hline Anthracothorax prevostii & res & no bos & 4 & cerco & 113 & 0.11 & 0.48 & 0.95 & 0.69 \\
\hline Dives dives & res & borde & 4 & pastizal & 112 & 0.08 & 3.88 & 4.25 & 4.87 \\
\hline Troglodytes aedon & res & no bos & 3 & cerco & 110 & & 3.24 & 6.18 & 2.95 \\
\hline Icterus prosthemelas & res & borde & 3 & cerco & 108 & & 0.06 & 0.34 & 0.16 \\
\hline Elaenia flavogaster & res & no bos & 3 & pastizal & 99 & & 3.78 & 3.14 & 5.91 \\
\hline Vermivora chrysoptera & mig & borde & 2 & cerco & 99 & 0.07 & & 3.76 & \\
\hline Sporophila aurita & res & no bos & 3 & pastizal & 94 & & 0.53 & 2.11 & 1.07 \\
\hline Dendroica coronata & mig & no bos & 1 & cerco & 93 & & & 0.53 & \\
\hline Myiarchus tyrannulus & res & no bos & 4 & pastizal & 91 & 0.18 & 1.16 & 1.71 & 3.28 \\
\hline Vireo flavifrons & mig & borde & 2 & pastizal & 91 & & 0.05 & & 0.07 \\
\hline Egretta caerulea & mig & no bos & 3 & pastizal & 89 & & 0.12 & 0.18 & 0.39 \\
\hline Coccyzus americanus & mig & no bos & 1 & cerco & 89 & & & 0.10 & \\
\hline Cotinga amabilis & res & interior & 1 & cerco & 89 & & & 0.10 & \\
\hline Passerina cyanea & mig & no bos & 3 & pastizal & 88 & & 0.11 & 0.21 & 0.31 \\
\hline
\end{tabular}




\begin{tabular}{|c|c|c|c|c|c|c|c|c|c|}
\hline ESPECIE & $\begin{array}{l}\text { CONDI- } \\
\text { CION }\end{array}$ & GRUPO & \#HABS & $\begin{array}{l}\text { Mayor } \\
\text { abund. }\end{array}$ & $\begin{array}{l}\text { DCA } \\
\text { EJE1 }\end{array}$ & $\begin{array}{l}\text { BOSQUE } \\
374 \pm 69 *\end{array}$ & $\begin{array}{c}\text { BOSQUE } \\
\text { RIBEREÑO } \\
199 \pm 25^{*}\end{array}$ & $\begin{array}{c}\text { CERCO } \\
\text { VIVO } \\
106 \pm 24^{*}\end{array}$ & $\begin{array}{c}\text { PASTIZAL } \\
16 \pm 54^{*}\end{array}$ \\
\hline Volatinia jacarina & res & no bos & 4 & pastizal & 87 & 0.15 & 0.54 & 0.96 & 1.06 \\
\hline Sporophila torqueola & res & no bos & 3 & pastizal & 84 & & 4.68 & 6.96 & 11.33 \\
\hline Camptostoma imberbe & res & no bos & 1 & pastizal & 76 & & & & 0.09 \\
\hline Quiscalus mexicanus & res & no bos & 3 & cerco & 75 & & 3.67 & 11.69 & 7.25 \\
\hline Columbina talpacoti & res & no bos & 2 & cerco & 73 & & & 1.54 & 0.44 \\
\hline Oryzoborus funereus & res & no bos & 3 & cerco & 64 & & 0.08 & 0.42 & 0.24 \\
\hline Charadrius vociferus & mig & no bos & 3 & pastizal & 45 & & 0.05 & 0.16 & 0.16 \\
\hline Caracara cheriway & res & no bos & 1 & pastizal & 0 & & & & 0.07 \\
\hline Jacana spinosa & res & no bos & 1 & pastizal & 0 & & & & 0.07 \\
\hline
\end{tabular}


